\title{
How to Intervene in the Root Caries Process? Systematic Review and Meta-Analyses
}

\author{
Hendrik Meyer-Lueckel ${ }^{a}$ Vita Machiulskiene ${ }^{b}$ Rodrigo A. Giacaman ${ }^{c}$ \\ ${ }^{a}$ Department of Restorative, Preventive and Pediatric Dentistry, University of Bern, Bern, Switzerland; ${ }^{\text {b } C l i n i c ~ o f ~}$ \\ Dental and Oral Pathology, Faculty of Odontology, Lithuanian University of Health Sciences, Kaunas, Lithuania; \\ ${ }^{c}$ Cariology Unit, Department of Oral Rehabilitation, University of Talca, Talca, Chile
}

\section{Keywords}

Root · Cervical · Caries · Carious · Composite · Glass ionomer cement · Fluoride $\cdot$ Chlorhexidine $\cdot$ Sealant $\cdot$ Minimum intervention · Oral health · Elderly

\begin{abstract}
The aim of this review as part of the preparation for a workshop organized by the European Federation of Conservative Dentistry (EFCD) in conjunction with the European Organisation for Caries Research (ORCA) was to systematically analyze available evidence of non-, micro- as well as invasive interventions for root caries lesions (RCLs). For each treatment strategy, a separate systematic review was either performed (micro-invasive and choice of restorative material) or updated (non-invasive and excavation technique) each of them following PRISMA guidelines, and if possible meta-analyses were performed. Besides the general advice to improve tooth brushing with fluoride toothpaste main findings for non-invasive interventions in RCLs, the use of dentifrices containing 5,000 $\mathrm{ppm} \mathrm{F}^{-}$as well as professionally applied chlorhexidine varnish or silver diamine fluoride seemed to be more efficacious to arrest root caries compared to conventional fluoride toothpaste or placebo respectively. However, this conclusion is based only on a few randomized clinical trials. For micro-invasive treatments, only 2 studies fo-
\end{abstract}

cusing on sealants were available without clear conclusions. A recent review on the comparison of atraumatic restorative treatment compared with conventional treatment concluded that there is insufficient data to clearly rule out if any difference with regard to restoration longevity between both techniques exists. When restoring coventionally, composites performed better than resin-modified and glass ionomer cements. However, all materials showed rather high annual failure rates in the majority of the studies and evidence is based on a low number of prospective studies with a rather high risk of bias.

(c) 2019 The Author(s) Published by S. Karger AG, Basel

\section{Introduction}

In restorative dentistry, numerous studies have been published with respect to direct invasive treatment of coronal caries. Meta-analyses show relatively high success and survival rates for restorations using amalgam and adhesively bonded materials in posterior teeth [Opdam et al., 2014; Schwendicke et al., 2016], but also for non-carious lesions in the cervical area [Santos et al., 2014; Schwendicke et al., 2016]. Nonetheless, for root caries lesions (RCLs), as discussed in a recent textbook, randomized clinical trials on lesion arrestment (i.e., non-invasive treat-

\begin{tabular}{|c|c|}
\hline KARGER & $\begin{array}{l}\text { (c) } 2019 \text { The Author(s) } \\
\text { Published by S. Karger AG, Basel }\end{array}$ \\
\hline $\begin{array}{l}\text { E-Mail karger@karger.com } \\
\text { www.karger.com/cre }\end{array}$ & $\begin{array}{l}\text { This article is licensed under the Creative Commons Attribution- } \\
\text { NonCommercial-NoDerivatives } 4.0 \text { International License (CC BY- } \\
\text { NC-ND) (http://www.karger.com/Services/OpenAccessLicense). } \\
\text { Usage and distribution for commercial purposes as well as any dis- } \\
\text { tribution of modified material requires written permission. }\end{array}$ \\
\hline
\end{tabular}

Prof. Dr. H. Meyer-Lückel

Department of Restorative, Preventive and Pediatric Dentistry zmk bern, Universität Bern, Freiburgstrasse 7 CH-3010 Bern (Switzerland)

E-Mail hendrik.meyer-lueckel@zmk.unibe.ch 
ment) as well as invasive intervention are scarce [Rocha de Olivera Carrilho, 2017]. Moreover, clinical evidence of the so-called lesion exposure method, laser therapy, resin infiltration or sealing, subsumed as micro-invasive strategies, has not been systematically analyzed so far.

In order to address the request of EFCD and ORCA to report evidence for treatment of RCLs at the highest possible level, we aimed for a systematic approach, if possible with additional meta-analyses. For non-invasive strategies, the authors together with the main reviewers (B.N. and S.P.) agreed upon updating a recent systematic review including a meta-analysis being co-written by one of the authors [Wierichs and Meyer-Lueckel, 2015]. However, unlike the former paper, we tried to focus on lesion arrest of RCLs rather than prevention of their initiation. For micro-invasive treatment strategies, no review could be retrieved from the dental literature, and thus a systematic approach had to be performed. With respect to the invasive treatments, the authors were aware of an initially unpublished manuscript [Göstemeyer et al., 2019] on the comparison of atraumatic restorative treatment (ART) with conventional treatment (CT) using glass ionomer cements (GICs) for restoring RCLs in seniors. This manuscript was adopted, but we additionally took into account evidence from younger age groups. Moreover, with regard to invasive treatment of RCLs, a recently published systematic review was revisited [Hayes et al., 2016]. Here one paper focusing on ART versus no ART had been included as well as a few others comparing the success of materials for restoration of RCLs, but no meta-analysis was attempted. The authors agreed on repeating the systematic research with respect to the choice of materials for invasive treatment of RCLs, first to check the inclusion of papers in the unpublished ART meta-analyses and second to perform, if possible, a meta-analysis on the success of various materials for restorative treatment of RCLs.

Several dimensions (factors) in the treatment of RCLs arose while preparing for the manuscript:

- Lesion severity: shallow or distinct cavity

- Lesion activity: yes or no

- Lesion extension: single or multiple surface or saucershaped (circled)

- Pulp involvement: yes or no

- Location: buccal (easily accessible for toothbrush), oral (more difficult to access), proximal (only accessible with interdental cleaning methods)

- Tooth: anterior, posterior

- Excavation technique: no (e.g., ART), selective (1-step vs. 2 step), non-selective (complete)
- Restorative material: GIC, resin-modified GIC (RMGIC), composite (Comp), others as compomers or various cements

- Technical issues: for example, use of matrices, application aids, and so on.

- Moisture control: Retraction chord and cotton rolls versus rubber dam

- Since we were aware to find only a few clinical studies regarding these factors, we tried to give guidance in a narrative way only whenever possible.

\section{Materials and Methods}

\section{General Review Design and Eligibility Criteria}

The reporting of this study is in accordance with PRISMA statement guidelines [Moher et al., 2009]. We followed PICO criteria with regard of how to intervene in the caries process in patients with RCL's non-, micro- or invasively with respect to lesion and patient characteristics with the outcome of lesion arrest, longevity of restorations or tooth survival, and, if possible, costs and quality of life, including randomized and quasi-randomized clinical trials, but also retrospective studies, if eligible.

Population:

Adults and seniors who retained a minimum of one natural tooth with exposed root surfaces without RCLs or with non-cavitated or cavitated RCLs also including patients with extreme conditions as xerostomia after irradiation on the head/neck area.

Interventions, controls and outcomes:

A: What is the evidence in order to prevent initiation of RCLs or to arrest non-cavitated or cavitated RCL's by non-invasive interventions as oral hygiene education, use of regular toothpaste alone or with the addition of remineralizing or antimicrobial compounds, 5,000 ppm fluoride toothpaste, fluoridated mouth rinses, silver diamine fluoride as well as fluoride gel, solution, fluid or varnish or chlorhexidine, ozone, polyols or probiotics?

$\mathrm{B}$ : What is the evidence in order to arrest non-cavitated or cavitated RCLs by micro-invasive methods as lesion exposure, lasers, resin infiltration or sealing?

C1: Is ART superior to CT using GICs with respect to the restoration of success and survival for cavitated RCLs comprising tooth integrity, aesthetics and pulp/pain prevention?

C2: What is the best material taking into account adhesive procedures (self-etch versus etch-rinse) with respect to the restoration of success and survival for cavitated RCLs in adults and seniors comprising tooth integrity, aesthetics and pulp/pain prevention?

\section{General Information Sources}

For each strategy with regard to invasivity, a separate systematic search was either updated (A), complemented with respect to younger age groups $(\mathrm{C} 1)$ or performed for this review (B and C2). For A and C1 PubMed, EMBASE and Cochrane Central Register of Controlled Trials (CENTRAL) were searched. For B and C2, PubMed was defined as being sufficient as a database. For all searches, titles and abstracts were independently screened for eligibility by 2 independent and calibrated reviewers (A: R.J.W. and H.M.L., update V.M. and H.M.L., B: R.A.G. and C.E.F., C1: G.G. 
and F.S., C2: L.S. and H.M.L.). Agreement concerning study inclusion or data extraction was achieved by discussion. Selected articles were screened full-text. Cross-referencing was performed to identify further articles to be assessed.

\section{Data Extraction in General}

For each of the reviews, data extraction was performed independently in duplicate. For longitudinal studies and clinical trials presented in different journals, only the most recent report of the study was taken. Unpublished data was not sought from authors (or obtained from other sources). Extracted data included:

- Study name, year of publication, study type location and setting (if not given, university setting was assumed)

- Participants (number, sex, and age)

- Characteristics of teeth and RCLs

- If possible, caries risk

- Mode of intervention or treatment including most relevant details also for controls

- Drop-outs, follow-up time, main outcome measures and findings

- Methodological issues (e.g., blinding if eligible, funding source for risk of bias assessment).

\section{Data Synthesis and Grading in General}

We refer to detailed descriptions within the published papers for search A [Wierichs and Meyer-Lueckel, 2015] and C1 [Göstemeyer et al., 2019]. For the search on restorative material comparison (C2) meta-analysis is described below. Here, no systematic grading was performed due to the low number of studies with rather high heterogeneity.

\section{Details for Non-Invasive Interventions (A)}

The search for non-invasive interventions in RCLs had recently (May 2014) been performed by the research group of one of the authors (H.M.L.) [Wierichs and Meyer-Lueckel, 2015] and was updated by VM together with HML for this review paper. For details regarding the original search strategy, review the paper directly. A similar strategy was performed as in 2014, but yielded no further suitable studies; thus a rather simple search with "root caries" as the term and "clinical trial" as the filter was additionally performed (Table 1). Analyses showed that various non-invasive interventions to prevent or arrest RCLs have been evaluated (online suppl. Table A1; for all online suppl. material, see www.karger.com/doi/10.1159/000501588), meta-analyses could only be performed for non-invasive interventions with similar outcome measures investigated in $>1$ study, but presentation of data will be restricted to those showing evidence at lower risk of bias.

\section{Details for Micro-Invasive Interventions (B)}

Due to the low number of eligible studies (online suppl. Table A3 and A7), meta-analysis could not be performed and results are reported narratively.

\section{Details for ART versus no ART (C1)}

As described by the authors, meta-analysis was performed for the outcome (risk of failure, measured as events per total restorations in each group) using Review Manager version 5.3. In our review, only per-protocol analysis is reported, but none of the intention-to-treat analyses [Göstemeyer et al., 2019].

Intervention Root Caries
Table 1. Search strategy as used for PubMed

Part A (non-invasive treatment [Wierichs and

Meyer-Lueckel, 2015])

Publication date from January 1, 1947 to May 31, 2014

Remineralization OR demineralization OR remineralisation OR demineralization OR texture OR hard* OR leathery OR soft* OR RCI OR root caries index OR dmf AND

Caries or carious AND

Root or cervic* OR dentin* OR tooth OR teeth

Part A (non-invasive treatment -update [1]) Filters: clinical trial Publication date from January 1, 2014 to February 28, 2019

Remineralization OR demineralization OR remineralisation OR demineralization OR texture OR hard* OR leathery OR soft* OR RCI OR root caries index OR dmf AND

Caries or carious AND

Root or cervic* OR dentin* OR tooth OR teeth

Plus search with term "root caries" filters: clinical trial (2)

Part B (micro-invasive treatment) filters: clinical trial No time restriction; date February 15, 2019

Lesion exposure OR laser OR infiltration OR sealant OR fluoride AND

Caries OR carious AND

Root OR cervic*

Part C1 (invasive treatment - ART vs. conventional therapy [Göstemeyer et al., 2019]) filters: clinical trial No time restriction; date; November 7, 2018

ART OR atraumatic OR restor* AND

Caries AND

Root

Part C2 (invasive treatment - conventional therapy

various materials) filters: clinical trial

No time restriction; date: February 15, 2019

Fill* OR restor* AND

Caries OR carious AND

Root or cervic*

RCI, Root Caries Index; ART, atraumatic restorative treatment. The asterisk indicates word stem search.

Details for Material Comparison for Invasive Treatment (C2)

With respect to intervention and control, we included studies using at least either one of the following restorative materials: GIC after non-selective excavation (i.e., no ART studies), RMGIC, Comp, amalgam and mineral trioxide. The outcome studied was restoration failure; possible reasons for failure in- 
Table 2. Publication and study search flow

\begin{tabular}{|c|c|c|c|c|c|c|}
\hline Records identified & 1,593 & 156 & 38 & 99 & 332 & 292 \\
\hline Full-text screened & 36 & 9 & 1 & 3 & 11 & 16 \\
\hline Hand-search & 16 & 0 & 0 & 0 & 0 & 7 \\
\hline Included in paper & 34 (30 studies) & 0 & 1 & 2 & 4 & 9 \\
\hline
\end{tabular}

\footnotetext{
* According to [Wierichs and Meyer-Lueckel, 2015].

\# For the update similar search (1) to the original strategy resulted in no suitable papers, but a second search (2) yielded to one paper being included.

ART, atraumatic restorative treatment; NA, not applicable.
}

cluded complete or partial retention loss, fracture, caries adjacent to the restoration, or other clinically and/or radiographically determined reasons for replacing or repairing the restoration.

Risk ratios and 95\% CIs were calculated for dichotomous outcome data (e.g., number of events per total restorations in each group) using Review Manager version 5.3 (The Cochrane Collaboration, 2014). Depending on the heterogeneity $\left(I^{2}<35 \%\right.$ or above), a fixed-effect model or a random-effect model was used to calculate a pooled estimate of effect (Mantel-Haenszel chi-square statistic [Mantel, 1963]). Heterogeneity was assessed using $I^{2}$ [Higgins and Thompson, 2002].

\section{Results}

In the recent search [Wierichs and Meyer-Lueckel, 2015] on the comparison of non-invasive agents, 1,593 articles were identified by screening electronic databases. Thirty-six articles were assessed for eligibility and 16 articles were identified by other sources (e.g., cross-references) (Table 2). A total of 52 articles were screened full-text and 18 articles were excluded (online suppl. Table A2). Originally, 34 articles reporting 30 trials with 10,136 patients, $20-101$ years of age were included, analyzing 28 different chemical agents (online suppl. Table A1). The median (25th/75th percentiles) follow-up time was $15(12 / 24)$ months. The updated search yielded 1 additional study, resulting in 11 studies investigating dentifrices, 10 rinses, 8 varnishes, 4 (fluoride) solutions, 3 gels, 2 ozone applications and 1 preventive dental regime. Eleven studies investigated both the initiation of RCLs and the activity change of RCLs, 12 analyzed the activity change of RCLs and 8 studies initiation of RCLs compared with baseline. The development of RCLs and their arrest were described most often using surface tex- ture (14 studies), decayed, missing, filled root surfaces (12 studies), electrical caries monitor (5 studies) or using the Root Caries Index (RCI) (4 studies). For details regarding dentifrices, mouth rinses, gels, ozone and the preventive dental regimes, we refer to the original paper [Wierichs and Meyer-Lueckel, 2015], since none of these agents revealed benefits with regard to the caries outcomes in comparison to standard therapy or control in studies with low risk of bias. Meta-analyses could not be performed for varnish containing 22,500 $\mathrm{ppm} \mathrm{F}^{-}$ (Duraphat), since it was used as a control group without a second (negative) control group [Fure and Lingstrom, 2009; Ravald and Birkhed, 1992] or the results were presented in a form that did not allowing meta-analyses [Schaeken et al., 1991; Tan et al., 2010]. However, fluoride varnish $\left(22,500 \mathrm{ppm} \mathrm{F}^{-}\right)$applied every 3 months seems to reduce the development of RCL more efficaciously [Schaeken et al., 1991; Tan et al., 2010] and arrested significantly more RCLs [Schaeken et al., 1991] compared when no additional agent was applied (online suppl. Table A6).

In our review, meta-analyses based on 6 trials with 1,210 patients completing the studies are presented (Fig. 1) for dentifrice containing $5,000 \mathrm{ppm} \mathrm{F}^{-}$, sodium diamine fluoride (SDF) or varnish containing chlorhexidine $(\mathrm{CHX})$ as adopted from the previous paper [Wierichs and Meyer-Lueckel, 2015] with no further update, but slight correction due to a mistake in the original presentation of the SDF data. Patients using dentifrice containing 5,000 $\mathrm{ppm} \mathrm{F}^{-}$(risk ratio [95\% CI] $=0.49$ [0.420.57]) [Baysan et al., 2001; Ekstrand et al., 2013] showed significantly higher numbers of RCLs being inactivated than patients using dentifrice containing 1,100-1,450 ppm $\mathrm{F}^{-}$at a low risk of bias. 


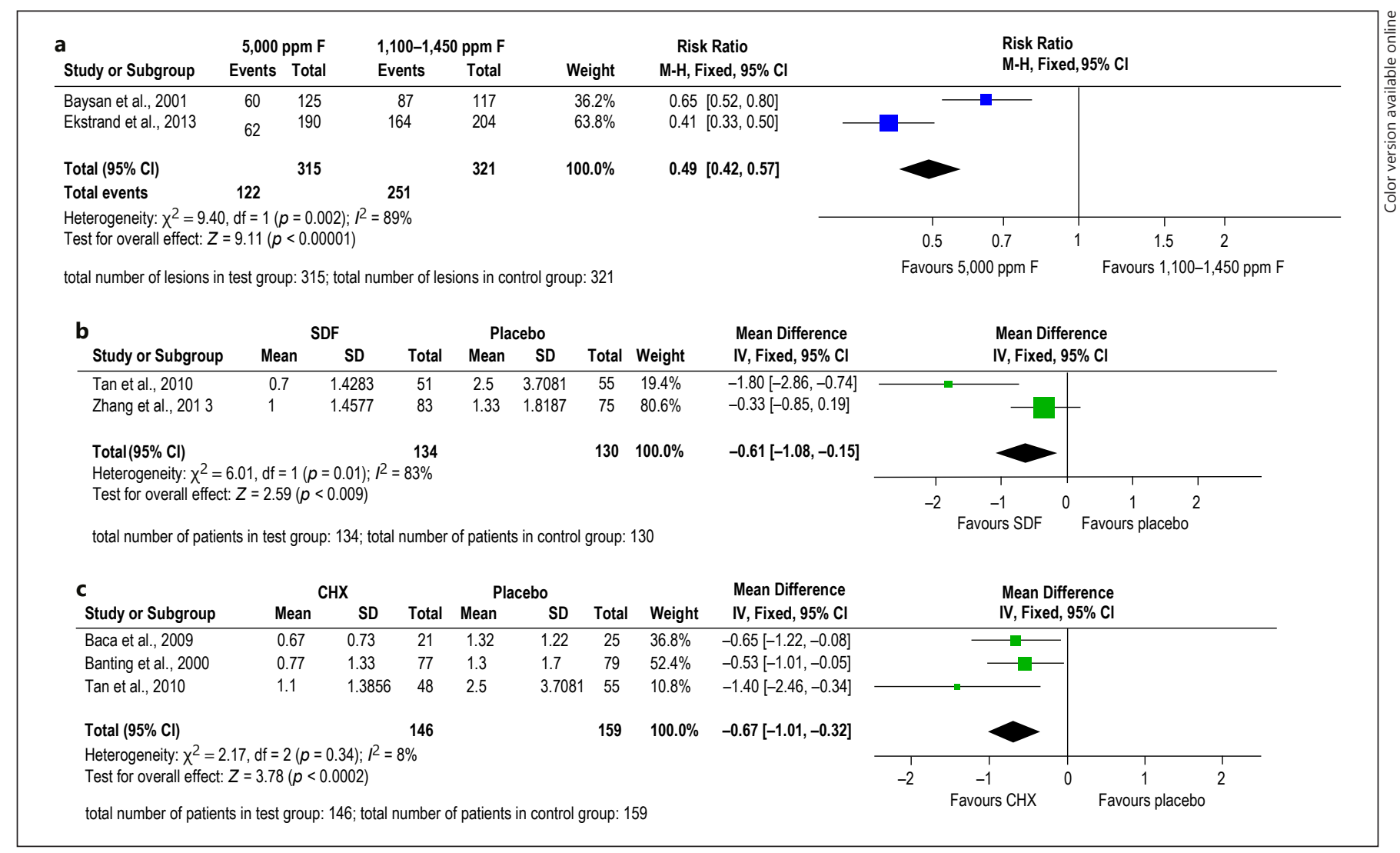

Fig. 1. a Forest plot of comparison: $5,000 \mathrm{ppm} \mathrm{F}^{-}$vs. 1,100-1,450 ppm $\mathrm{F}^{-}$dentifrice, for outcome: surface texture at 6-8 months follow-up time. b Forest plot of comparison: sodium diamine fluoride (SDF) vs. placebo varnish, for outcome: DMFRS (only new RCLs) at 24-36 months follow-up time. c Forest plot of comparison: chlorhexidine (CHX) vs. placebo varnish, for outcome: DMFRS (only new RCLs) at 21-36 months follow-up time. Updated

Of 11 studies investigating dentifrices, 2 studies used a non-fluoride dentifrice as a negative control. One of them using RCL initiation as an outcome [Jensen and Kohout, 1988 ] demonstrated a $67 \%$ difference in root caries incidence between test (dentifrice containing 1,100 $\mathrm{ppm} \mathrm{F}^{-}$) and control over 12 months. The other one showed twice (56 vs. $27 \%$, respectively, $p<0.001$ ) as much RCLs becoming harder in the test group (dentifrice containing $1,450 \mathrm{ppm} \mathrm{F}^{-}$) compared with the negative control within 6 months [Hu et al., 2013].

Professionally applied 1 or $10 \%$ CHX (adverse events reported) varnish (mean difference $[95 \% \mathrm{CI}]=-0.67$ [-1.01 to -0.32 ]) [Baca et al., 2009; Banting et al., 2000; Tan et al., 2010] as well as professionally applied SDF (mean difference $[95 \% \mathrm{CI}]=-0.61[-1.08$ to -0.15$]$ ) [Tan et al., 2010; Zhang et al., 2013] reduced the initiation of quantitative meta-analyses for use of 5,000 ppm toothpaste, SDF or CHX varnish against standard or placebo respectively adopted from [Wierichs and Meyer-Lueckel, 2015] and presented in a corrected form. decayed, missing, filled root surfaces or new RCLs were used to calculate mean differences and 95\% CIs for studies using the same units. Surface texture scores were used to calculate risk ratio and $95 \% \mathrm{CI}$.
RCLs significantly compared with placebo (Fig. 1), but with medium to high risk of bias. The newer study on SDF reported some benefits with respect to colour as a surrogate for inactivation in favour of SDF [Li et al., 2016] (online suppl. Table A6).

The focus of the preventive programs using oral hygiene instructions and/or dental education was observed in several studies, although only 1 clinical trial included a negative control group (no intervention) for comparison [Mojon et al., 1998]. A slight decrease of the number of teeth with active root caries (4.1\%) compared with $0.2 \%$ increase in the negative control group could be observed in the institutionalized elderly after 18 months. In another study, addressing a similar population of community dwelling elderly people [Zhang et al., 2013], an additional caries-inhibiting effect of the reinforced oral health ed- 


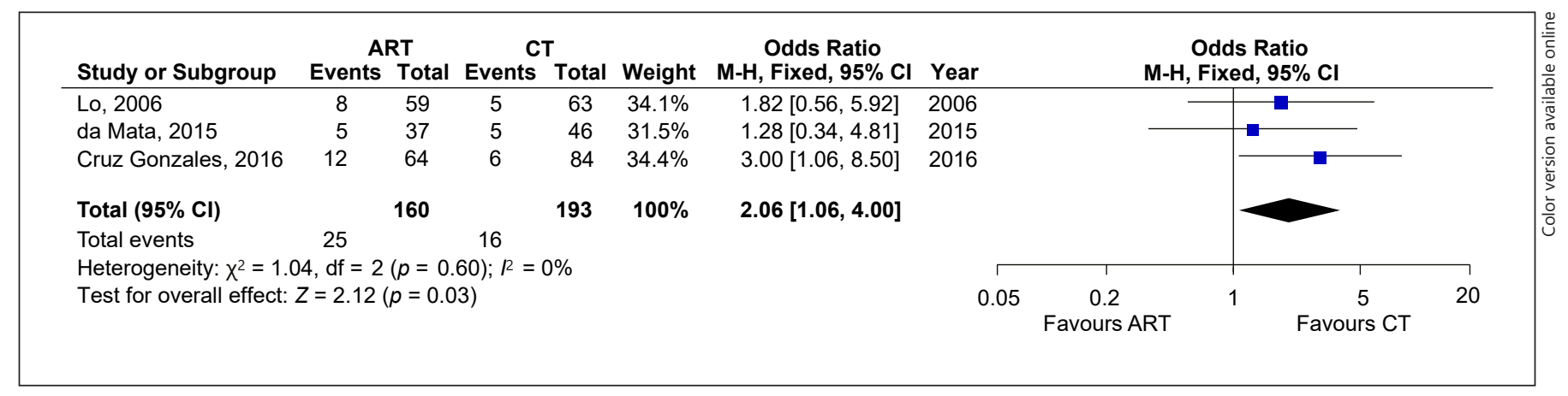

Fig. 2. Forest plots for the risk of failure of ART against CT both using GICs as reported recently [Göstemeyer et al., 2019]. OR and 95\% CIs are given. ART, atraumatic restorative treatment; CT, conventional treatment.

ucation to the annual application of 38\% SDF was estimated after 24 months for both the initiation and the activity control of the RCLs $(p<0.01)$.

The search for micro-invasive strategies for the management of RCLs retrieved 99 records. After title screening, 88 studies were excluded, and 11 records were assessed for eligibility by reviewing only the abstracts. Three records were selected for full-text reading and only 2 of them [Baysan and Lynch, 2007; Wicht et al., 2003] met the inclusion criteria (Table 2). The excluded study (online suppl. Table A3) [Rolland et al., 2011] measured antibacterial properties of a dentine bonding agent, but not a direct effect on RCLs. There was only one micro-invasive strategy identified in the selected studies; this was the use of sealants on RCLs. Studies on sealants with or without additional ozone application [Baysan and Lynch, 2007] or sealants with or without the additional use of amine fluoride [Wicht et al., 2003] were found. Sealant retention was low (ca. 46-67\%) [Baysan and Lynch, 2007] and no additional benefits by the use of the sealant compared with CHX varnish could be revealed [Wicht et al., 2003] (online suppl. Table A7).

According to the recently published meta-analysis regarding the comparison of ART versus CT, 235 studies were identified. Eleven of them screened full-text and 3 trials (Table 2) involving 130 patients (423 restorations) included (online suppl. Table A8). Excluded studies and reasons are given in the appendix (online suppl. Table A4). Risk of bias was high or moderate in all but one trial. ART was associated with a borderline significantly increased risk of failure (OR [95\% CI] 2.06 [1.06-4.00]; Fig. 2). The main reasons reported for failure were marginal defects or retention loss [da Mata et al., 2015; Lo et al., 2006]. In one trial, secondary caries was the main reason for failure [Cruz Gonzalez and Marin Zuluaga, 2016].
The annual failure was calculated from these data being ca. $15 \%$. One additional trial being excluded in the other review due to the younger age group showed similar success for both ART and CT [Hu et al., 2005].

Our search related to success and survival of restorations in RCLs resulted in 292 studies, of which 16 and additionally 7 found by hand-search were fully screened (Table 2). Excluded studies and reasons are given in the appendix (online suppl. Table A5). Since we omitted double reporting of an ART study [Lo et al., 2006] that was covered recently [Göstemeyer et al., 2019; Hayes et al., 2016], we included in addition to the 4 studies covered by the recent systematic review [Hayes et al., 2016] another 5 trials. This resulted in 9 studies, of which 7 were suitable for meta-analyses. Two studies could not be analysed either being the only one on the comparison of GIC with amalgam [Wood et al., 1993] or studying GIC only [Gilboa et al., 2012]. For the meta-analyses of GIC versus Comp (5 studies), GIC versus RMGIC and RMGIC versus Comp (both analyses 3 studies) all together 2,051 restorations in approximately 1,000 patients could be evaluated with a follow-up time of 12 months ( 1 study), 2 years (4) or approximately 3 years (2) (online suppl. Table A9). Meta-analyses revealed a significantly higher risk of failure for GIC (OR [95\% CI] 2.9 [1.1-7.7]; Fig. 3a) as well as RMGIC compared with Comp (OR [95\% CI] 2.5 [1.25.3]; Fig. 3b) but not between GIC and RMGIC (OR [95\% CI] 2.5 [0.8-8]) In particular, in irradiated patients, GIC showed higher failure rates compared with Comp (online suppl. Fig. A1). Annual failure rates for GIC and Comp were rather high in all prospective studies (ca. 44 and 17\% respectively), but much lower in the retrospective one (ca. $2.4 \%$ for both) [Wierichs et al., 2018]. Interestingly in the only study focusing on amalgam (29/35), much high retention rates could be revealed compared with GIC (3/35) 


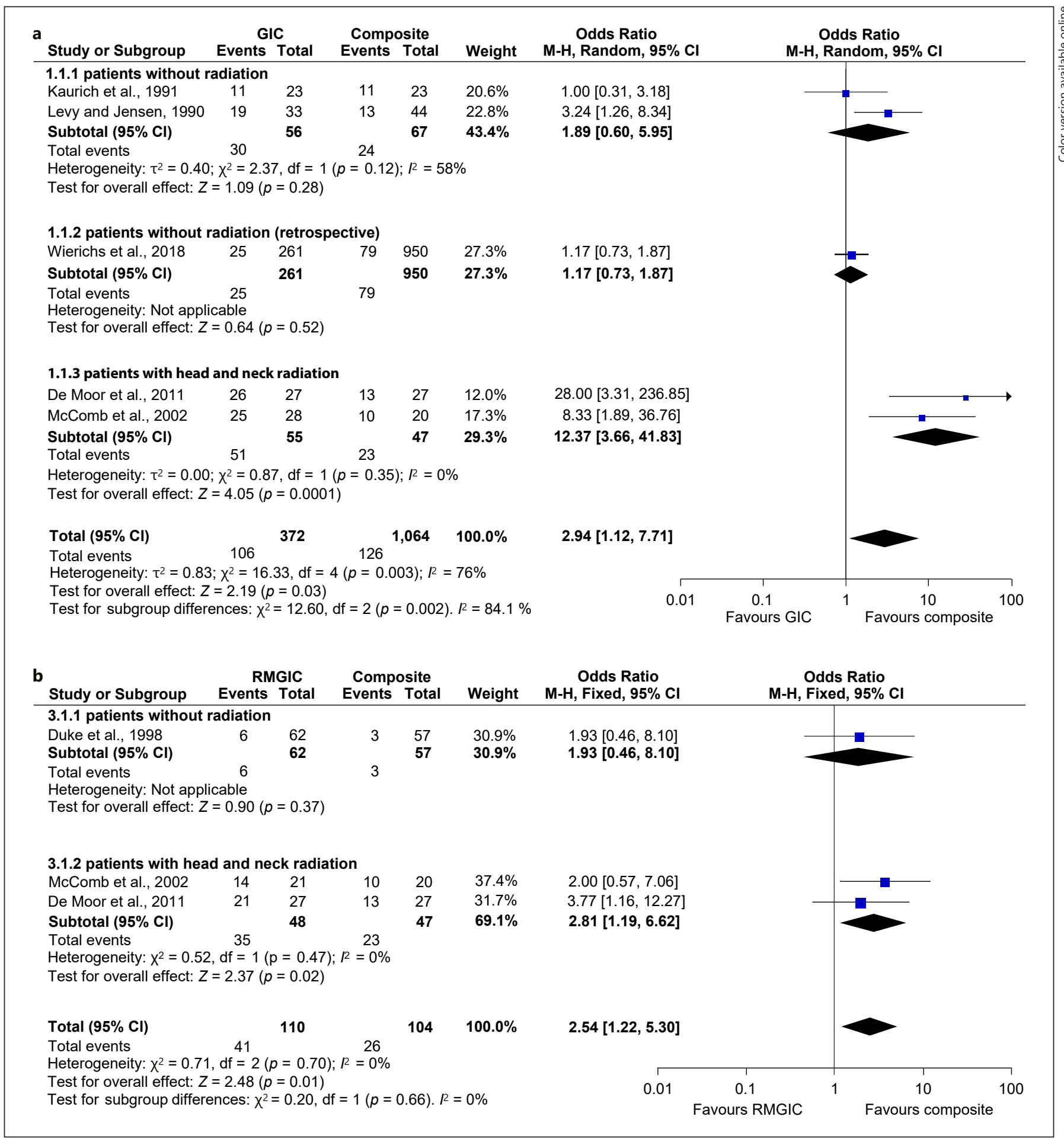

Fig. 3. Forest plots for the risk of failure of GIC versus Comp (a) as well as RMGIC versus Comp (b) in RCLs, taking into account study design and radiation of the head and neck area, showing significant higher ORs in favour for Comp. ORs and 95\% CIs are given. GIC, glass ionomer cement; RMGIC, resin-modified GIC. 
after 6 months [Wood et al., 1993]. A study dealing with longevity of GIC placed in interproximal RCLs showed a rather high survival rate (52/66) after a mean follow-up time of 39 months [Gilboa et al., 2012].

\section{Discussion}

This review aimed at summarizing the current evidence for the prevention and treatment (arrest and restoration) of RCLs. For non-invasive strategies, at least a few studies with lower risk of bias could be excerpted from the literature, which was not the case for micro-invasive approaches. For the invasive treatment, several clinical studies were found, but the focus of this review was shifted to the 2 research questions for which randomized clinical trials are available - the comparison of ART versus no ART and the choice of material.

Active root caries in cervical areas has been described as a rather fast process, leaving unsupported enamel coronally around the cement-enamel junction. This overhanging enamel promotes biofilm stagnation, so regular check-ups in order to re-motivate patients to perform oral hygiene properly seem to be the first choice to manage RCLs [Nyvad and Fejerskov, 1986]. Generally, all studies investigating dentifrices indicate that the RCLs can be controlled to a varying degree by brushing teeth with a regular fluoridated toothpaste. Fluoride acts as a therapeutic agent, in both, the lesion initiation, and in the lesion arrest mechanisms. However, in certain populations with compromised functional abilities in particular, the regular maintenance may not be sufficient. As recently summarized [Wierichs and MeyerLueckel, 2015] and in line with another review [Walsh et al., 2019], the benefits of fluoride toothpaste are dose-dependent, and thus the daily use of dentifrice containing $5,000 \mathrm{ppm} \mathrm{F}^{-}$could be advised based on its higher efficacy to control development and progression of RCLs.

Based on the results of the performed analyses, several other non-invasive measures might be suggested for the control of root surface caries. Although the use of CHXcontaining products (varnish or gel) in children and adolescents with regular exposure to fluoride led to inconclusive evidence [Twetman, 2004; Walsh et al., 2015], there could be some beneficial effect for RCLs [Wierichs and Meyer-Lueckel, 2015]. Recent reviews on SDF trials support its efficacy for root caries prevention and RCL arrest [McReinolds and Duane, 2018; Oliveira et al, 2018] in comparison with placebo and also with fluoride varnish [Oliveira et al, 2018]. However, studies on SDF were rated as being of high risk of bias [Wierichs and Meyer-Lueckel,
2015]. Nonetheless, regular applications of SDF to exposed root surfaces of older adults, due to their simplicity of use and cost-effectiveness [Schwendicke and Gostemeyer, 2017], might be considered an alternative measure to control RCLs, in particular, when it is not possible to perform regular dental care, for example, in a palliative setting.

It is important to note that cost effectiveness of different preventive methods needs to be considered alongside the clinical efficacy. Although the ultimate goal is to reduce the need for costly restorative treatments, the costs for certain non-operative measures may be higher than for the others, and not necessarily be equally effective. Thus, in the low caries risk populations, (with 16 teeth at risk), avoiding preventive treatment seemed to be the most cost-effective although providing least clinical effect, while in high caries-risk patients (with 24 teeth at risk), SDF applications were ranked as most cost-effective and less costly than the other non-operative interventions including "no treatment" [Schwendicke and Gostemeyer, 2017]. The present review has identified the evidence for a mild caries-inhibiting effect of the preventive dental education programs among the institutionalized elderly; however, this also needs to be weighed against the costs incurred by the implementation of such programmes.

In caries patients with high risk, non-invasive options may not be sufficient to arrest RCLs. Thus, various methods, such as the Ultraconservative Treatment [Baysan et al., 2001; Mijan et al., 2014], Non-Restorative Cavity Treatment or Non-Restorative Cavity Control (lesion exposure) [Gruythuysen, 2010; Hansen and Nyvad, 2017; van Strijp and van Loveren, 2018] have been discussed, as it has been described as being effective in primary teeth [Gruythuysen and van Strijp, 2018; Hansen and Nyvad, 2017]. Although lesion exposure has also been claimed to be efficacious for the management of RCLs [Gruythuysen and van Strijp, 2018], no controlled trials could be found.

Root sealants may be another option to stabilize cervical carious areas [Baysan and Lynch, 2007; Wicht et al., 2003]. However, no differences in root surface texture could be revealed between the application of CHX-varnishes and sealants [Srinivasan et al., 2013; Wicht et al., 2003]. These results should be interpreted with caution, since sealants covering root lesions may have interfered with the direct assessment of texture and fluorescence readings. In conclusion, due to the very limited evidence for the use of sealants in root caries, no clinical recommendations can be made.

No compelling evidence to support either ART or CT for restoring RCLs in older adults could be found [Göstemeyer et al., 2019], also when taking into account one 
additional study with younger patients being irradiated in the head/neck area [Hu et al., 2005]. However, based on the limited number of included trials, CT may be more efficacious than ART for this purpose. Interestingly for GIC annual failure rates, where only one third compared to the results in the prospective studies, the current metaanalysis on material comparisons was included. However, the retrospective, practice-based study revealed very low failure rates, which might be explained partially by the design, that is, patients with failures might not have attended the follow-ups, but also the better general condition of the patients [Wierichs et al., 2018].

Regarding the various materials to restore RCLs, rather high failure rates compared with those for coronal direct restorations [Opdam et al., 2014; Schwendicke et al., 2016] have been reported. This might be explained by the high-risk groups (xerostomic patients after irradiation) included in many of the studies on RCLs, and also due to the more difficult circumstances (for example, location, moisture control, age, bad oral hygiene). Interestingly enough, both the study on interproximal GIC restorations [Gilboa et al., 2012] as well as the retrospective study including 50\% 2-surface RCLs and mostly Comp as the restorative material [Wierichs et al., 2018] in more general population settings showed rather promising (low) failure rates. Nonetheless, we may corroborate the conclusion of the recent systematic review [Hayes et al., 2016] including half of the studies as in our current review that more number of well-performed randomized clinical trials in more generalizable cohorts are needed to give evidence-based recommendations.

\section{Acknowledgement}

The authors are indebted to C.E.F. from the University of Talca in Chile for screening the titles of the search of micro-invasive strategies to treat RCLs, extracting and helping in the interpretation of the data. The authors are also thankful to L.S. from University of Bern in Switzerland for screening the titles and abstracts for inclusion and exclusion of suitable papers with regard to various materials for invasive treatment of RCLs as well extracting data for online supplementary Table A8. We also like to thank R.J.W. from RWTH Aachen University in Germany for performing the metaanalyses for those studies focusing on various materials for invasive treatment of RCLs. Moreover, we sincerely thank G.G., F.S. and co-authors for providing their manuscript before acceptance as well as data for Figure 2. Finally, we like to thank the 2 reviewers of this paper and leaders of the respective group for caries treatment in seniors at the consensus meeting in Berlin, B.N. (Aarhus, Denmark) and S.P. (Berlin, Germany) for their valuable support and comments.

\section{Disclosure Statement}

The authors declare that they have no conflicts of interest to disclose.

\section{Author Contributions}

H.M.-L., V.M., and R.A.G.: designed and planned the search strategies in consultation with B.N. and S.P., H.M.-L., V.M., R.A.G., L.S., and C.E.F.: identified studies from PubMed and screened for eligibility and inclusion. Meta-analyses of non-invasive interventions (A) and material comparisons for invasive treatments (C2) were performed by R.J.W. H.M.L. wrote a first draft of the manuscript that was complemented and revised by V.M. and R.A.G.

\section{References}

Baca P, Clavero J, Baca AP, González-Rodríguez MP, Bravo M, Valderrama MJ. Effect of chlorhexidine-thymol varnish on root caries in a geriatric population: a randomized double-blind clinical trial. J Dent. 2009 Sep;37(9): 679-85.

Banting DW, Papas A, Clark DC, Proskin HM, Schultz M, Perry R. The effectiveness of $10 \%$ chlorhexidine varnish treatment on dental caries incidence in adults with dry mouth. Gerodontology. 2000 Dec;17(2):6776.

Baysan A, Lynch E, Ellwood R, Davies R, Petersson L, Borsboom P. Reversal of primary root caries using dentifrices containing 5,000 and 1,100 ppm fluoride. Caries Res. 2001 Jan-Feb; 35(1):41-6.

Baysan A, Lynch E. Clinical reversal of root caries using ozone: 6-month results. Am J Dent. 2007 Aug;20(4):203-8.
Cruz Gonzalez AC, Marín Zuluaga DJ. Clinical outcome of root caries restorations using ART and rotary techniques in institutionalized elders. Braz Oral Res. 2016 May;30(1): 30.

da Mata C, Allen PF, McKenna G, Cronin M, O'Mahony D, Woods N. Two-year survival of ART restorations placed in elderly patients: A randomised controlled clinical trial. J Dent. 2015 Apr;43(4):405-11.

De Moor RJ, Stassen IG, van 't Veldt Y, Torbeyns D, Hommez GM. Two-year clinical performance of glass ionomer and resin composite restorations in xerostomic head- and neckirradiated cancer patients. Clin Oral Investig. 2011 Feb;15(1):31-8.

Duke ES, Robbins JW, Snyder DS. Clinical evaluation of a dentinal adhesive system: threeyear results. Quintessence Int. 1991 Nov; 22(11):889-95.
Ekstrand KR, Poulsen JE, Hede B, Twetman S, Qvist V, Ellwood RP. A randomized clinical trial of the anti-caries efficacy of 5,000 compared to $1,450 \mathrm{ppm}$ fluoridated toothpaste on root caries lesions in elderly disabled nursing home residents. Caries Res. 2013;47(5):391-8.

Fure S, Lingström P. Evaluation of different fluoride treatments of initial root carious lesions in vivo. Oral Health Prev Dent. 2009;7(2):147-54.

Gilboa I, Cardash HS, Baharav H, Demko CA, Teich ST. A longitudinal study of the survival of interproximal root caries lesions restored with glass ionomer cement via a minimally invasive approach. Gen Dent. 2012 Jul-Aug; 60(4):e224-30.

Göstemeyer G, da Mata C, McKenna G, Schwendicke F. Atraumatic vs conventional restorative treatment for root caries lesions in older patients: Meta- and trial sequential analysis. Gerodontology. 2019 May;ger.12409. 
Gruythuysen RJ, van Strijp AJ. [Preventive dentistry 9. Non-Restorative Cavity Treatment: advanced insight or controversial?]. Ned Tijdschr Tandheelkd. 2018 Jan;125(1):33-41.

Gruythuysen RJ. [Non-Restorative Cavity Treatment. Managing rather than masking caries activity]. Ned Tijdschr Tandheelkd. 2010 Mar;117(3):173-80.

Hansen NV, Nyvad B. Non-operative control of cavitated approximal caries lesions in primary molars: a prospective evaluation of cases. J Oral Rehabil. 2017 Jul;44(7):537-44.

Hayes M, Brady P, Burke FM, Allen PF. Failure rates of class $\mathrm{V}$ restorations in the management of root caries in adults - a systematic review. Gerodontology. 2016 Sep;33(3):299307.

Higgins JP, Thompson SG. Quantifying heterogeneity in a meta-analysis. Stat Med. 2002 Jun; 21(11):1539-58.

Hu DY, Yin W, Li X, Feng Y, Zhang YP, Cummins $\mathrm{D}$, et al. A clinical investigation of the efficacy of a dentifrice containing $1.5 \%$ arginine and $1450 \mathrm{ppm}$ fluoride, as sodium monofluorophosphate in a calcium base, on primary root caries. J Clin Dent. 2013;24 Spec no A:A23-31.

Hu JY, Chen XC, Li YQ, Smales RJ, Yip KH. Radiation-induced root surface caries restored with glass-ionomer cement placed in conventional and ART cavity preparations: results at two years. Aust Dent J. 2005 Sep;50(3):18690.

Jensen ME, Kohout F. The effect of a fluoridated dentifrice on root and coronal caries in an older adult population. J Am Dent Assoc. 1988 Dec;117(7):829-32.

Kaurich M, Kawakami K, Perez P, Munn T, Hasse AL, Garrett NR. A clinical comparison of a glass ionomer cement and a microfilled composite resin in restoring root caries: two-year results. Gen Dent. 1991 Sep-Oct;39(5):346-9.

Levy SM, Jensen ME. A clinical evaluation of the restoration of root surface caries. Spec Care Dentist. 1990 Sep-Oct;10(5):156-60.

Li R, Lo EC, Liu BY, Wong MC, Chu CH. Randomized clinical trial on arresting dental root caries through silver diammine fluoride applications in community-dwelling elders. J Dent. 2016 Aug;51:15-20.

Lo EC, Luo Y, Tan HP, Dyson JE, Corbet EF. ART and conventional root restorations in elders after 12 months. J Dent Res. 2006 Oct;85(10): 929-32.

Mantel N. Chi-Square Tests with One Degree of Freedom; Extensions of the Mantel-Haenszel
Procedure. J Am Stat Assoc. 1963;58:690700.

McComb D, Erickson RL, Maxymiw WG, Wood RE. A clinical comparison of glass ionomer, resin-modified glass ionomer and resin composite restorations in the treatment of cervical caries in xerostomic head and neck radiation patients. Oper Dent. 2002 Sep-Oct;27(5):430-7.

Mijan M, de Amorim RG, Leal SC, Mulder J, Oliveira L, Creugers NH, et al. The 3.5-year survival rates of primary molars treated according to three treatment protocols: a controlled clinical trial. Clin Oral Investig. 2014 May; 18(4):1061-9.

Moher D, Liberati A, Tetzlaff J, Altman DG; PRISMA Group. Preferred reporting items for systematic reviews and meta-analyses: the PRISMA statement. PLoS Med. 2009 Jul; 6(7):e1000097.

Mojon P, Rentsch A, Budtz-Jørgensen E, Baehni PC. Effects of an oral health program on selected clinical parameters and salivary bacteria in a long-term care facility. Eur J Oral Sci. 1998 Aug;106(4):827-34.

Nyvad B, Fejerskov O. Active root surface caries converted into inactive caries as a response to oral hygiene. Scand J Dent Res. 1986 Jun; 94(3):281-4.

Opdam NJ, van de Sande FH, Bronkhorst E, Cenci MS, Bottenberg P, Pallesen U, et al. Longevity of posterior composite restorations: a systematic review and meta-analysis. J Dent Res. 2014 Oct;93(10):943-9.

Ravald N, Birkhed D. Prediction of root caries in periodontally treated patients maintained with different fluoride programmes. Caries Res. 1992;26(6):450-8.

Rocha de Olivera Carrilho M. Root Caries: From Prevalence to Therapy. In: Lussi A, Buzalaf MA, editors. Monographs in Oral Science. Basel: Karger; 2017.

Rolland SL, McCabe JF, Imazato S, Walls AW. A randomised trial comparing the antibacterial effects of dentine primers against bacteria in natural root caries. Caries Res. 2011;45(6): $574-80$.

Santos MJ, Ari N, Steele S, Costella J, Banting D. Retention of tooth-colored restorations in non-carious cervical lesions-a systematic review. Clin Oral Investig. 2014;18(5):1369-81.

Schaeken MJ, Keltjens HM, Van Der Hoeven JS. Effects of fluoride and chlorhexidine on the microflora of dental root surfaces and progression of root-surface caries. J Dent Res. 1991 Feb;70(2):150-3.
Schwendicke F, Göstemeyer G, Blunck U, Paris S, Hsu LY, Tu YK. Directly Placed Restorative Materials: Review and Network Meta-analysis. J Dent Res. 2016 Jun;95(6):613-22.

Schwendicke F, Göstemeyer G. Cost-effectiveness of root caries preventive treatments. J Dent. 2017 Jan;56:58-64.

Srinivasan M, Schimmel M, Riesen M, Ilgner A, Wicht MJ, Warncke M, et al. High-fluoride toothpaste: a multicenter randomized controlled trial in adults. Community Dent Oral Epidemiol. 2014 Aug;42(4):333-40.

Tan HP, Lo EC, Dyson JE, Luo Y, Corbet EF. A randomized trial on root caries prevention in elders. J Dent Res. 2010 Oct;89(10):1086-90.

Twetman S. Antimicrobials in future caries control? A review with special reference to chlorhexidine treatment. Caries Res. 2004 May-Jun;38(3):223-9.

van Strijp G, van Loveren C. No Removal and Inactivation of Carious Tissue: Non-Restorative Cavity Control. Monogr Oral Sci. 2018;27: 124-36.

Walsh T, Oliveira-Neto JM, Moore D. Chlorhexidine treatment for the prevention of dental caries in children and adolescents. Cochrane Database Syst Rev. 2015 Apr;(4):CD008457.

Walsh T, Worthington HV, Glenny AM, Marinho VC, Jeroncic A. Fluoride toothpastes of different concentrations for preventing dental caries. Cochrane Database Syst Rev. 2019 Mar;3:CD007868.

Wicht MJ, Haak R, Lummert D, Noack MJ. Treatment of root caries lesions with chlorhexidine-containing varnishes and dentin sealants. Am J Dent. 2003 Sep;16(Spec No):25A30.

Wierichs RJ, Kramer EJ, Meyer-Lueckel H. Risk factors for failure of class $\mathrm{V}$ restorations of carious cervical lesions in general dental practices. J Dent. 2018 Oct;77:87-92.

Wierichs RJ, Meyer-Lueckel H. Systematic review on noninvasive treatment of root caries lesions. J Dent Res. 2015 Feb;94(2):261-71.

Wood RE, Maxymiw WG, McComb D. A clinical comparison of glass ionomer (polyalkenoate) and silver amalgam restorations in the treatment of Class 5 caries in xerostomic head and neck cancer patients. Oper Dent. 1993 MayJun;18(3):94-102.

Zhang W, McGrath C, Lo EC, Li JY. Silver diamine fluoride and education to prevent and arrest root caries among communitydwelling elders. Caries Res. 2013;47(4):28490. 